supports the validity of option B + as the national PMTCT standard in Ethiopia.

\section{P6.064 INTEGRATING SIMULTANEOUS TRIPLE POINT-OF-CARE SCREENING FOR SYPHILIS, HEPATITIS B AND HIV IN ANTENATAL SERVICES THROUGH RURAL OUTREACH TEAMS IN GUATEMALA}

doi:10.1136/sextrans-2013-051184.1217

${ }^{1} \mathrm{~A}$ Smith, ${ }^{2} \mathrm{M}$ Sabidó, ${ }^{1} \mathrm{E}$ Camey, ${ }^{3} \mathrm{~A}$ Batres, ${ }^{2} \mathbf{J}$ Casabona. ${ }^{1}$ Fundacion Sida i Societat, Escuintla, Guatemala; ${ }^{2}$ Fundacion Sida i Societat, Barcelona, Spain; ${ }^{3}$ Escuintla Health Department, MSPAS, Escuintla, Guatemala

Background In May 2012, a simultaneous triple point-of-care screening programme for syphilis, hepatitis B (HBV) and HIV was integrated in antenatal services in rural Guatemala. The programme included screening for pregnant women with mobile outreach teams, promotion through community networks, partner notification, and linkage to care. Our aim was to evaluate its feasibility and effectiveness in increasing testing uptake, case detection and referrals for positive cases.

Methods A network of over 200 midwives and community volunteers promoted testing and recruited pregnant women through community IEC campaigns. Nurses from three mobile outreach teams, two health posts, and the district health centre offered counselling and triple screening by fingerprick. Syphilis cases were treated on-site. HIV and HBV were referred with accompaniment to the tertiary hospital for care and prevention of mother to child transmission. Testing uptake was compared with the 8-month period prior to implementation of the programme.

Results In 8 months, 978 women sought prenatal services; 65\% were screened for HIV and syphilis and $62 \%$ for HBV. Testing uptake increased 209\%/30\% from baseline for HIV/syphilis ( $p<0.001$ ). $29 \%$ were screened during the first trimester. Six cases of syphilis were detected and treated (0.95\%); two cases of HIV (0.32\%) were detected and initiated prophylaxis/treatment; and 0 cases of HBV. All of these cases were identified by the rural outreach teams or health posts. Seven women notified their partners (5 for syphilis, 2 for HIV) yet only 1 partner was tested, resulting positive. Three children were born with suspected congenital syphilis and all three died.

Conclusions This outreach programme shows the feasibility of simultaneous screening for three infections in rural Guatemala and its effectiveness in increasing screening coverage, case detection, and access to treatment services. Further efforts should work to improve earlier detection, results of partner notification, and adverse outcomes due to congenital syphilis.

\section{P6.065 SEXUAL HEALTH IN GERMANY - USING INDICATORS AS AN INSTRUMENT TO DESCRIBE, PLAN AND EVALUATE. A CATALOGUE OF INDICATORS OF THE GERMAN STI SOCIETY}

doi:10.1136/sextrans-2013-051184.1218

\begin{abstract}
${ }^{1,2} \mathbf{K}$ Jansen, ${ }^{1,2} \mathrm{~V}$ Bremer, ${ }^{3,2} \mathrm{U}$ von Rüden, ${ }^{4,2} \mathrm{E}$ Steffan, ${ }^{5,2 \mathrm{H}}$ Nitschke. ${ }^{1}$ Robert KochInstitute, Berlin, Germany; ${ }^{2}$ German STI Society, Bochum, Germany; ${ }^{32}$. Federal Centre for Health Education, Cologne, Germany; ${ }^{4}$ SPI Research, Berlin, Germany; ${ }^{5} 4$. HIV and STI Counseling and Testing Centre of the Cologne Health Department, Cologne, Germany
\end{abstract}

Background Sexual health is an integral part of the personal wellbeing of human beings, as defined by the WHO. Thus, instruments to measure the respective needs, to design appropriate and effective interventions, and to evaluate their impact and success are of high importance. These tools should be adopted on a local, regional and national level. There have been no such instruments available to specify the situation of sexual health in Germany to date. The "Sexual Health" section of the German STI Society has now adopted a set of indicators in order to depict the sexual health status of people in Germany, as well as to plan and evaluate activities for the improvement of sexual health as a whole.

Methods Basis for defining indicators consisted of draught submittals of WHO and EU. All indicators described were considered as to their explanatory power and applicability for the specific German situation in terms of political structures, medical care, and epidemiology, and were partly adopted, revised or complemented by new indicators. Every indicator was operationalised in terms of appropriate variables, type of documentation, administration level, data source, availability, and assessed by its specific importance.

Results 39 indicators on policy and social determinants, 28 on access (availability, information, demand, and quality), 24 on the use of services, and 33 outcome/impact indicators were defined. To allow for flexibility, these categories were grouped by the following subdivisions: general, family planning, maternal and prenatal health, STI/RTI/reproductive morbidities, promotion of sexual health, adolescent sexual education and health, and sexual violence.

Conclusion A logical, convincingly conclusive and yet manageable list of indicators for Germany has been compiled. The selected indicators display the German situation and are at the same time highly comparable internationally. Potential users of the document are health experts, policy developers, researchers and other health care professionals.

\section{P6.066 WHERE ARE WE NOW AND WHERE ARE WE GOING?: A META-ANALYSIS OF 10 YEARS OF SEXUAL HEALTH SERVICE INTEGRATION IN GLASGOW, SCOTLAND, UK}

doi:10.1136/sextrans-2013-051184.1219

1,2R Ilett. 'Sandyford Sexual Health Services, NHS Greater Glasgow and Clyde, Glasgow, UK; '2University of Glasgow, Glasgow, UK

Background In 2000, family planning and reproductive health, genitourinary medicine, and women's health converged to form integrated sexual health services in Glasgow and Clyde, Scotland the first large-scale UK example. The service now supports 100, 000 people annually via 250 multi-skilled staff in 20 sites across a large post-industrial area with major deprivation, and $1+$ million population. Its services include contraception, termination, HIV and STI testing, young people's and gay men's services, counselling and a public library. After ten years, the service commissioned three external evaluations to assess success and potential developments. This paper describes a meta-analysis of these.

Methods The evaluations used qualitative and quantitative methods including reviewing activity; undertaking staff surveys, user engagement, stakeholder interviews, population research and feedback from doctors and health professionals. The meta-analysis systematically reviewed the evaluations to triangulate main themes, patterns and disconnects.

Results The meta-analysis revealed significant themes. For example, only $5 \%$ of local young people knew the service at its start and $61 \%$ a decade later, whereas people in their 40s did not think it relevant. Strategically, the service was felt to have developed an innovative resource for professionals and policy-makers and to be leading sexual health developments and training nationally, but not engaging with local health care enough.

Conclusion The meta-analysis reveals a service that has demystified sexual health care and influenced nationally and more widely The paper concludes with recommendations that the service should remain integrated, with inequalities-sensitive practise central, and continue inclusive approaches to staff involvement. The service should better utilise social media; engage more with other health staff; better understand older people's motivations to use services; focus more on men, especially young men, and young people from 
deprived and minority ethnic communities; maintain its vision to deliver and promote integrated services, and be flexible in possible developments.

\section{P6.067 FIVE YEAR SURVIVAL OF ADULT HIV-INFECTED PATIENTS INITIATED ON HAART AT THE AIDS SUPPORT ORGANISATION(TASO), UGANDA}

doi:10.1136/sextrans-2013-051184.1220

SSALI L, F Wasagaami, J Birungi, C Bakanda. The AIDS Support Organization, TASO, Kampala, Uganda

Background The AIDS Support Organization (TASO) Antiretroviral Program was rolled out in November 2004. The study aimed to assess adherence status of adult patients surviving for 60 months after HAART initiation in a resource poor setting.

Methodology Retrospective cohort study of adult patients who initiated HAART 60 months prior to the date of data abstraction at TASO Soroti, Eastern Uganda. Participants included in the study were male and female adult ( $\geq 14$ years) patients who initiated HAART at TASO Soroti (between $1^{\text {st }}$ August 2005 to $31^{\text {st }}$ March 2012). Since 2005 TASO Soroti has been providing HAART free of charge to patients either at World Health Organization (WHO) stage III or IV irrespective of CD4 cell count, or at any WHO stage with CD4 cell counts $\leq 350$ cells/ $\mu 1$.

Results Of the 4827 adults patients included in the analysis, $32.1 \%$ were male and the median age was 43 years. $67.9 \%$ were female and the median age was 41 years. $94.9 \%$ of patient were HAART naïve, $5.1 \%$ were transfer in. 3913(81.1\%) of 4827 patients were still on HAART after 5 years of follow-up. 18.9\% difference is attributed to lost to follow-up, patient transferred out and reported deaths. Among the patients with adherence assessment reported, $96.6 \%$ of patients had adherence level $>95 \%, 1.5 \%$ had adherence level of $85 \%-95 \%$ and $1 \%$ had adherence $<85 \% .74 .5 \%$ patients received their HAART refills at the CDDP, $23.8 \%$ received refills at the facility, and $1.4 \%$ received refills at their homes.

Conclusions These positive results after 5 years of initiating HAART in patients with advanced HIV disease demonstrate efficacy of HAART in resource-limited settings. Additional support is required to ensure timely HAART among adults.

\section{P6.068 EFFECT OF AN HIV PREVENTION PROGRAM AMONG FEMALE SEX WORKERS ON THE DECLINE IN SYPHILIS PREVALENCE AMONG THEIR CLIENTS IN SOUTH INDIA}

doi:10.1136/sextrans-2013-051184.1221

'RAJARAM S, ${ }^{2} \mathrm{M}$ Alary, ${ }^{3 P}$ Banandur, ${ }^{4} \mathrm{U}$ K Thammattoor, ${ }^{4} \mathrm{~T}$ Thomas, ${ }^{5} \mathrm{M}$ K Mainkar, ${ }^{5} \mathrm{R}$ Paranjape, ${ }^{6} \mathrm{R}$ Adhikary, ${ }^{2} \mathrm{~T}$ Duchesne, ' $\mathrm{S}$ Isac. ${ }^{1}$ Karnataka Health Promotion Trust, Bangalore, India; ${ }^{2}$ Centre de recherche, CHU de Québec, Québec, OC, Canada, ${ }^{3}$ National Institute of Mental Health and Neurosciences, Bangalore, India; ${ }^{4}$ St John's Research Institute, Bangalore, India; ${ }^{5} \mathrm{National}$ AIDS Research Institute, Pune, India; ${ }^{6} \mathrm{FHI}$ 360, Washington DC, WA, United States

Objective As a part of evaluating the Avahan Programme, two rounds of integrated biological and behavioural assessment (IBBA) surveys were conducted in 2006-07 and 2009-10 among clients of female sex workers (FSWs) across 17 districts in south India to measure the prevalence of HIV and STIs, and related risk factors ( $n \sim 7,000$ per round). We examined the association between changes in programme indicators and changes in syphilis prevalence.

Methods Using a random-effect multilevel logistic regression, we examined the effect of district-specific programme indicators for FSWs (from the Avahan computerised monitoring system) on the decline in syphilis prevalence among clients of FSWs after controlling for significant individual level variables. Program indicators included their 2006 value, the difference in their values between the surveys, and the interaction between the latter and study round. The model also controlled for baseline syphilis prevalence and its interaction with survey rounds.

Results Syphilis prevalence among clients of FSWs decreased from $4.8 \%$ (round 1 ) to $2.6 \%$ (round $2 ; \mathrm{p}<0.001$ ). The odds ratio (OR) of the interaction term between the difference in programme coverage (\% of FSWs covered by the programme in a given year) and the round was 0.983 ( $p=0.001)$, indicating that increase in coverage was significantly associated with the decrease in syphilis prevalence between rounds. ORs comparing syphilis prevalence between rounds varied with the level of increase in coverage and were statistically significant with coverage increase $\geq$ quartile $(\mathrm{Q}) 1: \mathrm{OR}=0.61$ at $\mathrm{Q} 1,0.53$ at $\mathrm{Q} 2,0.40$ at $\mathrm{Q} 3$ and 0.13 at $\mathrm{Q} 4$.

Conclusions The results support that the Avahan intervention among FSWs had a significant impact in reducing syphilis prevalence among their clients in settings covered by the intervention The triangulation of our results with those obtained among FSWs suggests a major impact of this intervention on the HIV/STI epidemic in southern India.

\section{P6.069 SOCIAL MOBILIZATION AND PEER-MENTORING TO ENCOURAGE VOLUNTARY COUNSELING AND TESTING AND POST-TESTING BEHAVIOR: A RANDOMIZED EXPERIMENT IN SENEGAL}

doi:10.1136/sextrans-2013-051184.1222

1J Graff Zivin, ${ }^{2}$ V Orozco, ${ }^{3} \mathrm{H}$ Thirumurthy, ${ }^{4} \mathrm{C}$ Sakho, ${ }^{5 \mathrm{P}}$ Diallo, ${ }^{2 \mathrm{M}}$ Offer Westort. ${ }^{1}$ University of California - San Diego, San Diego, CA, United States; 'World Bank, Washington, DC, United States; ${ }^{3}$ University of North Carolina - Chapel Hill, Chapel Hill, NC, United States; ${ }^{4}$ Ministry of Health, Dakar, Senegal; ${ }^{5}$ National AIDS Commission, Dakar, Senegal

Background To evaluate the effectiveness of two HIV/AIDS sensitization campaigns of the community-based organisations (CBOs) it financed, the National AIDS Commission of Senegal randomly assigned its financial support across the country's 14 regions, establishing three groups of health districts. Health districts where NACfunded $\mathrm{CBO}$ s carried out i) mass social mobilisation communication campaigns; ii) campaigns using peer-mentoring; and iii) control health districts, where CBOs didn't receive NAC support.

Methods We exploit the initial random assignment and differences at the time of implementation for the intervention groups (the randomization plan was not followed in the control group so we remove it from the analysis). We use two methods. First, a beforeafter analysis where we examine trends to estimate the change in outcomes during each of the four quarters following implementation of the intervention, relative to the average of the four quarters before the intervention. Second, we estimate a difference-in-differences regression model to directly compare the effectiveness of each intervention.

Results The before-after analysis shows that immediately after the intervention was completed, peer-mentoring doubled the number of adults who were tested for HIV and the number of adults who picked up their test results. For both outcomes, the number tripled three quarters later. Social mobilisation was less effective over time. For those individuals who test HIV-positive, peer-mentoring induces more HIV-infected individuals to collect their results and engage in post-test counselling. However, the second method finds no significant differences between the two intervention groups.

Conclusion These results suggest that both interventions are successful for the general population and to a lesser for the HIV-infected population. The difference-in-differences analysis suggests that there is little reason to favour one intervention over the other. Analysis at the health district level greatly diminishes our power to detect changes, especially for social mobilisation activities. 